\title{
Pair Interaction of Dislocations in Two-Dimensional Crystals
}

\author{
C. Eisenmann, U. Gasser, P. Keim, and G. Maret \\ Universität Konstanz, 78457 Konstanz, Germany \\ H. H. von Grünberg \\ Karl-Franzens-Universität, $8010 \mathrm{Graz}$, Austria \\ (Received 18 April 2005; published 26 October 2005)
}

\begin{abstract}
The pair interaction between crystal dislocations is systematically explored by analyzing particle trajectories of two-dimensional colloidal crystals measured by video microscopy. The resulting pair energies are compared to Monte Carlo data and to predictions derived from the standard Hamiltonian of the elastic theory of dislocations. Good agreement is found with respect to the distance and temperature dependence of the interaction potential, but not regarding the angle dependence where discrete lattice effects become important. Our results on the whole confirm that the dislocation Hamiltonian allows a quantitative understanding of the formation and interaction energies of dislocations in two-dimensional crystals.
\end{abstract}

DOI: $10.1103 /$ PhysRevLett.95.185502

PACS numbers: 61.72.Ff, 61.72.Lk, 64.70.Dv

The elastic theory of dislocations is at the heart of modern crystal physics and plays a pivotal role in determining fundamental crystal properties, such as, for instance, their growth and mechanical properties, formation of grain boundaries or internal stresses, or the fatigue behavior of crystals. The central idea of this theory was introduced by Taylor [1], one of their pioneers, and refers to the notion that dislocations can interact with one another as particles in a dilute gas. Taylor assumed that the force between two dislocations can be calculated from the stress field produced from one dislocation acting on an element of the dislocation line of the other, an assumption that was later justified more rigorously by Eshelby [2]. This then made it possible to set up a dislocation interaction Hamiltonian which, in its most general form, consists of terms for the screw and edge components of the dislocation [3].

This Letter reports on an experimental study testing quantitatively the predictions of Taylor's dislocation interaction Hamiltonian, thus extending previous work [4] in which a full quantitative analysis was not possible. Our idea is to investigate topological defects of crystals made of colloidal particles, having a diameter as large as a few $\mu \mathrm{m}$. Particles of such a size can be directly observed under the microscope and conveniently tracked over many hours by appropriate video microscopy techniques. With the trajectories of all the crystal particles at hand, one can readily compute the probabilities of the formation of certain defect pairs, which subsequently allows one to estimate the defect interaction energies.

We examine 2D crystals where dislocations are no longer defect lines as in three dimensions, but rather point defects characterized by Burgers vectors $\vec{b}(\vec{r})$ which are dimensionless Bravais lattice vectors of the 2D crystal lattice. Since in 2D screw dislocations cannot exist, the Hamiltonian, which we experimentally test here, reduces to the interaction energy for an isolated pair of edge dis- locations at $\vec{r}$ and $\vec{r}^{\prime}$,

$$
\begin{aligned}
\beta H_{D}= & -\frac{K}{4 \pi}\left[\vec{b}(\vec{r}) \cdot \vec{b}^{\prime}\left(\vec{r}^{\prime}\right) \log \frac{R}{a}-\frac{[\vec{b}(\vec{r}) \cdot \vec{R}]\left[\vec{b}^{\prime}\left(\vec{r}^{\prime}\right) \cdot \vec{R}\right]}{R^{2}}\right] \\
& +2 \beta E_{c},
\end{aligned}
$$

with $\vec{R}=\vec{r}-\vec{r}^{\prime}$ and $\beta=1 / k_{B} T$. The coupling constant $K$ is related to the Lamé elastic constants $\mu$ and $\lambda, K=$ $4 \beta \mu a_{0}^{2}(\mu+\lambda) /(2 \mu+\lambda) ; E_{c}$ is the core energy, i.e., the energy needed for the creation of an isolated dislocation, while $a$ is the dislocation core diameter to be distinguished from $a_{0}$, the lattice constant of the underlying lattice (a triangular lattice in our case).

Our experiment has already been described in a number of publications [5-7], the technical data of the specific setup used here are the same as in [7]. Therefore, we restrict ourselves to just summarizing the essentials here. Spherical colloids of a diameter of $d=4.5 \mu \mathrm{m}$ are confined by gravity to the air-water interface of a hanging water droplet. With a bending of the interface smaller than half a micron over the field of view $(835 \times 620 \mu \mathrm{m})$, our colloid system can be considered as the realization of an almost perfect 2D system. Coordinates of about 2500 particles (out of $2.5 \times 10^{5}$ particles of the whole sample) at equal time steps (every $500 \mathrm{~ms}$ ) were recorded with an accuracy better than $0.1 \mu \mathrm{m}$ using digital video microscopy. Averages, when given in the following, were typically taken over 2500 configurations (in Fig. 3 only each tenth configuration was analyzed). To check the statistics, we also computed averages on the basis of only the first half of all configurations. The colloidal particles are superparamagnetic due to $\mathrm{Fe}_{2} \mathrm{O}_{3}$ doping. A magnetic field $\vec{B}$ applied perpendicular to the air-water interface induces in each particle a magnetic moment $\vec{M}=\chi \vec{B}$, which leads to a repulsive dipole-dipole pair interaction potential $\beta v(r)=\Gamma /(\sqrt{\pi \rho} r)^{3}$ with the dimensionless interaction 
strength parameter $\Gamma=\beta\left(\mu_{0} / 4 \pi\right)(\chi B)^{2}(\pi \rho)^{3 / 2}$, with $\rho=2 /\left(\sqrt{3} a_{0}^{2}\right)$ being the $2 \mathrm{D}$ area density. Thus, the colloidal interaction is not only precisely known, but can also be externally tuned by varying the magnetic field $B$. $\Gamma$ is determined as described in Ref. [6]; it may be thought of as being an inverse system temperature and is the only parameter controlling the phase behavior of the system. For $\Gamma>\Gamma_{m}$ the sample is a hexagonal crystal; the inverse melting temperature $\Gamma_{m}=60$ was determined in [6] by means of the relative Lindemann parameter and the decay behavior of the translational order parameter. The Lamé elastic constants for this crystal-in its ideal form as a hexagonal, defect free lattice at $T=0$-are also known, and with these constants the coupling constant becomes $K=1.258 \Gamma$ [7]. Finally, the core energy $E_{c}$ can be estimated with the help of the renormalization-flow diagram of the theory of 2D melting [8]. We find $\beta E_{c}=1.788 K / 8 \pi$ [9]. We have also carried out accompanying Monte Carlo (MC) simulations using the $\Gamma / r^{3}$ pair potential for 3600 crystal particles (periodic boundary conditions, cutoff of $5 a_{0}, 50000 \mathrm{MC}$ cycles).

The quantity we actually measure is the number of dislocations per frame. A dislocation can be identified by finding a fivefold coordinated colloid being the nearest neighbor to a sevenfold coordinated particle, where the number of nearest neighbors of each colloid is determined by means of a Voronoi construction. The Burgers vector characterizing this dislocation is a lattice vector of unit length perpendicular to the line joining both particles [10]. We take the midpoint of this line to define the center of the dislocation. The centers of two such dislocations are connected by the distance vector $\vec{R}$; see inset of Fig. 1(b). Figure 1 shows particle coordination plots of typical configurations from experimental data in both the crystalline (a) and the liquid phase (b). For $\Gamma \gg \Gamma_{m}$ the overwhelming majority of dislocations occur in form of bound dislocation pairs with antiparallel Burgers vectors, which from time to time appear, dissociate, recombine again, and finally vanish, as exemplified in Fig. 1(c). It is clear from this figure how local distortions of particles on a regular hexagonal lattice can lead to the formation of a dislocation pair at minimum distance: four nearest neighbor particles of a hexagonal lattice have five nearest neighbor bonds of length $a_{0}$ and one next-nearest neighbor bond of length $\sqrt{3} a_{0}$. These four particles can be continuously shifted such that a rectangle is formed with side lengths $a_{0}$ and $\sqrt{3} a_{0} / 2$ and a diagonal $\sqrt{7} a_{0} / 2$. Two particles are then fivefold coordinated, and the other two sevenfold coordinated, and a dislocation pair has formed. The distance $R_{77}$ between the two sevenfold coordinated particles now is $\sqrt{7} a_{0} / 2$. The right inset of Fig. 2 shows the vector $\vec{R}_{77}$ of all these minimum-distance dislocation pairs: indeed, $R_{77}$ equals $\sqrt{7} a_{0} / 2$. The plot also reveals that these pairs have no particular preference for any of the three main directions of the hexagonal lattice. In the following, we therefore need not specify the direction of the individual Burgers vectors, but only the angle $\theta$ between $\vec{R}$ and $\vec{b}$. A $(\theta, R)$ plot of the minimum-distance dislocation pairs is given in the left inset of Fig. 2 with the corresponding $\theta$ histogram in the main figure. Since $\theta<0$ implies disappearance of dislocations because $R_{77}$ becomes too large for the two sevenfold coordinated particles to remain nearest neighbors [see Fig. 1(b)], the histogram shows a sharp edge for $\theta<0$, and the whole distribution becomes asymmetric as there is no such sharp limitation for pairs with positive $\theta$. This angle distribution offers the first possibility to test Eq. (1). If $R$ equals the minimum distance $a$ and $\vec{b}=-\vec{b}^{\prime}$, then Eq. (1) reduces to $\beta H_{D}=-K /(4 \pi) \cos ^{2} \theta$, and $\exp \left(-\beta H_{D}\right)$ becomes $\exp \left[1.258 \Gamma /(4 \pi) \cos ^{2} \theta\right]$, plotted as a solid line in Fig. 2. The experimental distribution is observed to be much narrower than the theoretical one, a
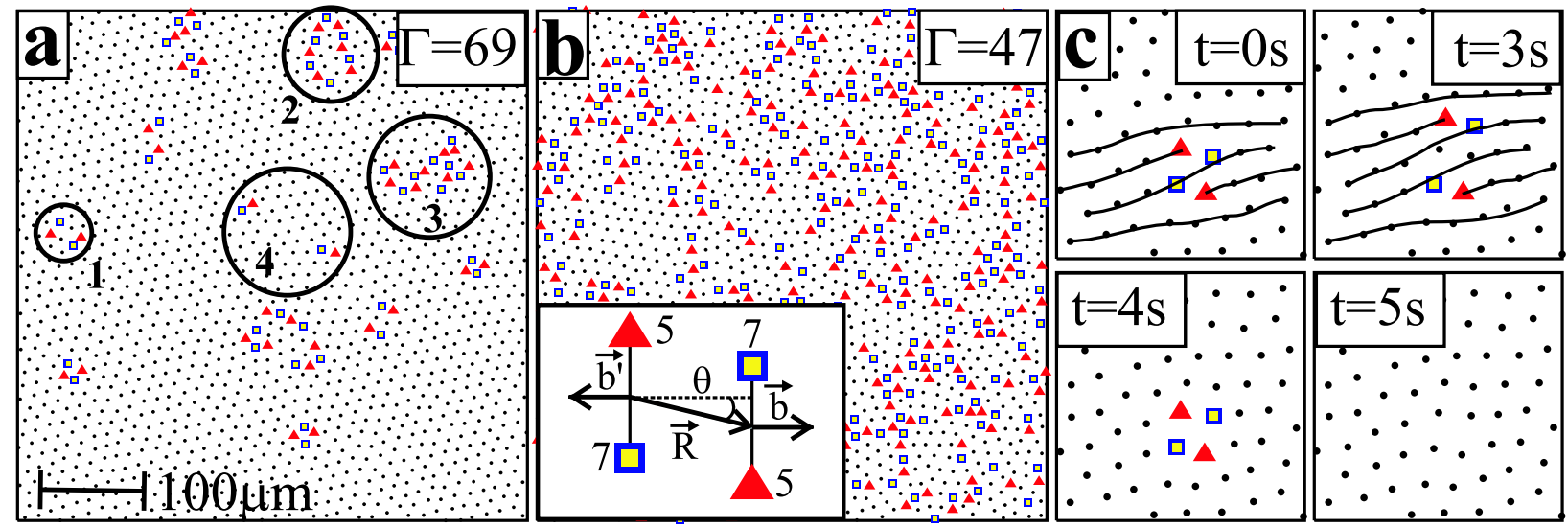

FIG. 1 (color online). Experimental particle configurations in the crystalline (a) and liquid phase (b) in which particles with 5 and 7 neighbors are highlighted by triangles and squares, respectively, while sixfold coordinated particles are plotted as dots. Panel (c) shows the dynamics of a typical dislocation pair: Within several seconds the pair dissociates into two dislocations, recombines again, and vanishes. The solid lines indicate the lattice lines, two of them ending at the fivefold coordinated particles. 


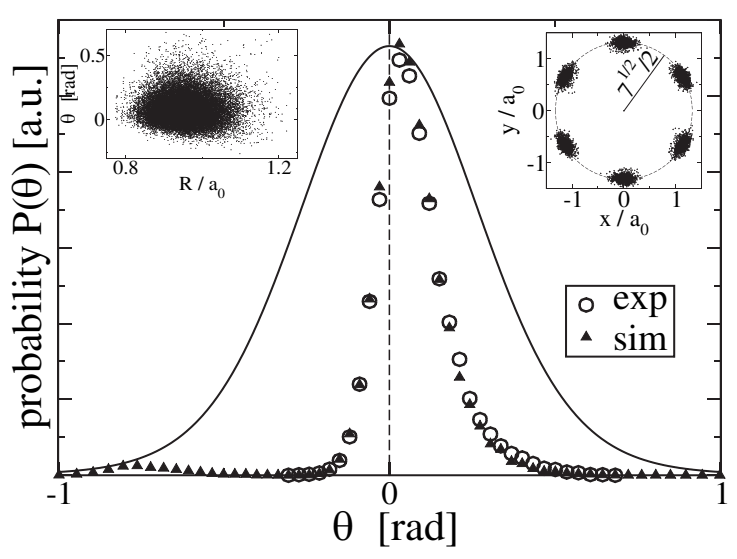

FIG. 2. Distribution of angles between the Burgers vector $\vec{b}$ and the distance vector $\vec{R}$ of dislocation pairs in their ground state $\left(R \approx a, \vec{b}=-\vec{b}^{\prime}\right)$. Symbols, experiment and simulation as indicated; solid line, prediction according to Eq. (1). Insets as explained in the text. $\Gamma=69$.

disagreement that arises from a failure of continuum theory which Eq. (1) is based on and which by its nature is expected to work on larger length scales only. Moreover, Eq. (1) ignores the hard-core interaction between the particles and steric effects are thus not taken into account. To explain the experimental angle distribution one would have to compute the energy landscape of the distorted discrete lattice around dislocation pairs fixed at $(R, \theta)$ by allowing the elastic stress in the neighborhood of the pair to relax. More direct is the MC simulation which reproduce the experimental curve almost perfectly as our figure shows.

We next explore the $R$ dependence of Eq. (1). For each dislocation with Burgers vector $\vec{b}$, we search for all partners with an antiparallel Burgers vector leading to an $R$ distribution as in the inset of Fig. 3. In order to compare our results with the theory we compute dislocation pair probabilities $P(R)$ by dividing the number of pairs $E(R) \Delta R$ within the distance range $R-\Delta R / 2$ and $R+$ $\Delta R / 2$ by the number of possible pairs, $3 N N_{1}(R) \Delta R$ on a lattice with $N$ sites $\left[N_{1}(R) \Delta R=2,12,18,24\right.$ for shell $R_{1}$ to $R_{4}$ with shell thickness $\Delta R=0.9 a_{0}$ ] [11]. Figure 3 shows $-\log [P(R)] / \Gamma$ resulting from analyzing both the $\mathrm{MC}$ and the experimental configurations. Assuming $\cos \theta \approx 1$, which according to the finding of Fig. 2 should be a good approximation (at least for the minimumdistance pairs), Eq. (1) for a dislocation pair with $\vec{b}=$ $-\vec{b}^{\prime}$ simplifies to

$$
-\log P(R)=\beta H_{D}=\frac{K}{4 \pi}\left[\log \frac{R}{a}+0.788\right],
$$

with $K=1.258 \Gamma$ and the distance $\sqrt{3} a_{0} / 2=0.87 a_{0}$ between two lattice lines taken as an estimate of the dislocation core diameter $a$. Figure 3 compares $-\log P(R) / \Gamma$ of Eq. (2) with the experimental and simulational data. Good agreement is found considering that no parameter has been

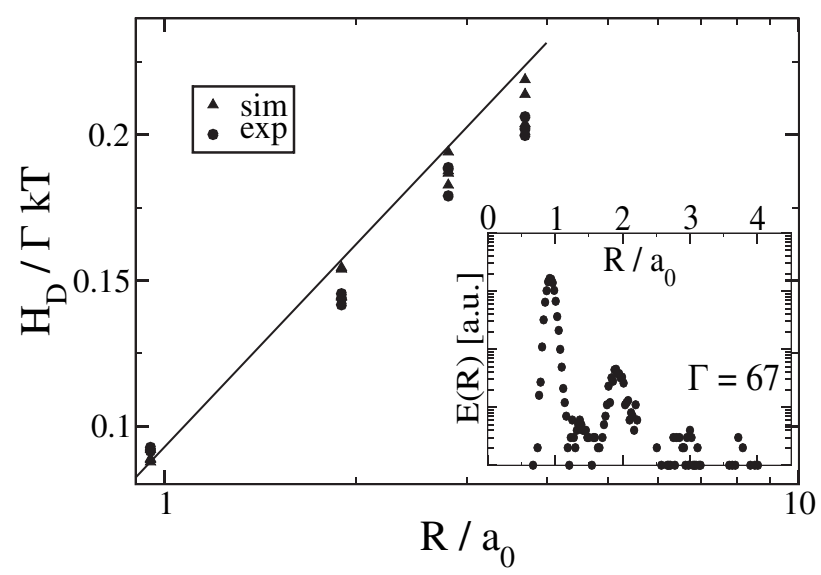

FIG. 3. Distance dependence of the pair interaction energy $-\log P(R)=\beta H_{D}(R)$ of dislocation pairs with antiparallel Burgers vectors. Circles, experiment $(\Gamma=65,67,69)$; solid triangles, simulation $(\Gamma=61,64,70,75)$; solid line, prediction according to Eq. (1). Note the logarithmic scale of the $R$ axis in the main figure. As shown in the inset, the $R$ distribution exhibits peaks, which is a signature of the underlying crystal lattice.

fitted. The remaining deviations can be understood as follows. Defect pairs such as those in circle 1 in Fig. 1(a) can be interpreted unambiguously in terms of Eq. (1); larger defect clusters, however, cannot (circles 2 and 3 in Fig. 1). While in case of the defect cluster in circle 2, it is still possible to identify three dislocation pairs, each with antiparallel Burgers vectors, it is probably not correct to neglect higher order interactions by naively assuming that the interaction energy of this cluster is given by sums of pair potentials as in (1). Clusters such as in circle 3 present another special case: here one can identify three dislocations whose Burgers vectors add up to $\overrightarrow{0}$. Such so-called higher complexions cannot be analyzed in terms of Eq. (1) and are omitted. However, higher order complexions, such as four-dislocation clusters, are not that simple to identify and are often erroneously counted. An additional source of error is the zero temperature approximation of the Young's modulus which at finite temperature is expected to slightly differ from its $T=0$ value. Finally, there are experimental artifacts such as the two dislocations in circle 4 which turn out to be absolutely immobile and are probably simply caused by dust particles or defective colloid particles. All these factors lead necessarily to a defect probability that for larger $R$ is higher, and thus to interaction energies that are lower, than predicted by Eq. (2). The differences between the simulation and the experimental data can also be taken as an estimate of the importance of finite size effects, as there are a 100 times more particle used in the experiment than in the simulation.

Figure 4 investigates the temperature dependence of the dislocation pair interaction energy in Eq. (1): $-\log P\left(R_{1}\right)$ of the minimum-distance pairs is plotted versus $\Gamma$. As is to 


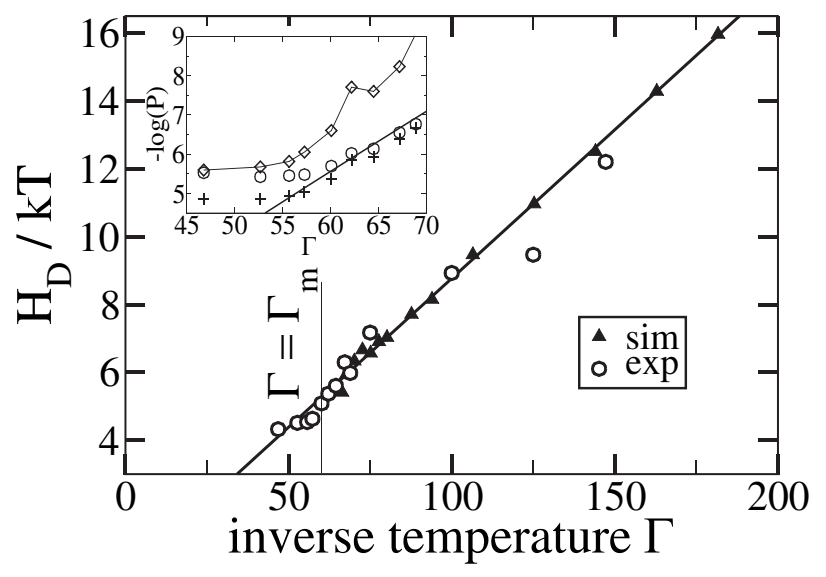

FIG. 4. Temperature dependence of the pair interaction energy $-\log P=\beta H_{D}$ of minimum-distance dislocation pairs $(R=$ $\left.0.95 a_{0}, \vec{b}=-\vec{b}^{\prime}\right)$. Symbols as in previous figures; solid line, prediction according to Eq. (1). Melting transition at $\Gamma_{m}=60$. Inset: $-\log P$ of isolated dislocations, $P=P_{57}$ (circles), pairs of isolated disclinations, $P=P_{5 / 7}$ (diamonds), the sum of both $P=$ $P_{57}+P_{5 / 7}$ (crosses), and the theoretical curve derived from Eq. (1).

be seen in Fig. 3, $R_{1}=0.95 a_{0}$, and Eq. (2) becomes $1.258 \Gamma / 4 \pi\left[\log \left(R_{1} / 0.87 a_{0}\right)+0.788\right]$, plotted as a solid line in Fig. 4. We find again good agreement for both the experimental and the simulational data, in case of the experiment even below $\Gamma_{m}=60$ where the system is no longer crystalline and where Eq. (1) ceases to make sense.

The Hamiltonian in Eq. (1) reaps additional significance from the important role it plays in the Kosterlitz-ThoulessHalperin-Nelson-Young theory of two-dimensional melting [8] where it defines the starting point for the derivation of the renormalization procedure for the elastic constants [12]. According to this theory, the translational symmetry of the crystal is destroyed by the dissociation of dislocation pairs, while dissociation of dislocations into isolated disclinations (fivefold and sevenfold coordinated particles having exclusively sixfold coordinated neighbors) destroys the orientational order. The statistics of both, isolated dislocations and disclinations, is studied in the inset of Fig. 4. The figure shows the probabilities $P_{5 \mid 7}=\left(E_{5}+E_{7}\right) /$ $(2 N)$ and $P_{57}=E_{57} /(3 N)$ and the sum of both probabilities, where $E_{5}, E_{7}$ is the number of fivefold and sevenfold coordinated particles, having exclusively sixfold coordinated neighbors, and $E_{57}$ is the number of isolated dislocations, understood here simply as the $5 / 7$ pairs that are surrounded only by sixfold coordinated particles. The solid curve is $\sum 2 P(R) N_{1}(R)$ with $P(R)$ from Eq. (2) and the sum running over $R=2 a, 3 a, 4 a$. It is evident that Eq. (2) provides the correct description for values of $\Gamma$ down to 55 for the sum $P_{5 \mid 7}+P_{57}$, but not for the probability $P_{57}$ of isolated dislocations that we actually measure. For this probability, deviations from the theoretical curve start becoming observable for $\Gamma<\Gamma_{m}$ since now a growing number of dislocations vanish by dissociating into isolated disclinations. Note also the change of the differences in the formation energies: While deep in the crystalline phase (at $\Gamma=70$ ), more than $10 \mathrm{kT}$ is required for the formation of an isolated disclination, but only $7 \mathrm{kT}$ for an isolated dislocation, both defect types becoming equally probable at higher temperatures, well below $\Gamma_{m}(5.5 \mathrm{kT}$ at $\Gamma=55)$.

In conclusion, we systematically explored the mutual attraction between dislocations in 2D crystals. Our results show that, except for its angle dependence, the Hamiltonian in Eq. (1) provides an adequate and quantitatively correct description of the pair interaction of dislocations in two-dimensional colloidal crystals. It has been successfully tested for temperatures up to the melting temperature and dislocation pair distances as small as one lattice constant.

We would like to thank K. Zahn, who initiated our interest in the topic of the present work. We also thank D. Reinke for his help with the MC code, and J. Zanghellini for a careful reading of the manuscript. This work has been supported by the DFG by means of the SFB TR 6 .

[1] G. I. Taylor, Proc. R. Soc. A 145, 362 (1934).

[2] J. D. Eshelby, Phil. Trans. R. Soc. A 244, 87 (1951).

[3] F. R. N. Nabarro, Theory of Crystal Dislocations (Dover, New York, 1987).

[4] A. Pertsinidis and X. S. Ling, Phys. Rev. Lett. 87, 098303 (2001); P. Schall, I. Cohen, D. A. Weitz, and F. Spaepen, Science 305, 1944 (2004).

[5] K. Zahn, J. M. Mendez-Alcaraz, and G. Maret, Phys. Rev. Lett. 79, 175 (1997); P. Keim, G. Maret, U. Herz, and H. H. von Grünberg, Phys. Rev. Lett. 92, 215504 (2004).

[6] K. Zahn, R. Lenke, and G. Maret, Phys. Rev. Lett. 82, 2721 (1999).

[7] H. H. von Grünberg, P. Keim, K. Zahn, and G. Maret, Phys. Rev. Lett. 93, 255703 (2004).

[8] D. R. Nelson and B. I. Halperin, Phys. Rev. B 19, 2457 (1979).

[9] We have searched for an $E_{c}$ such that the crossing of the separatrix and the line of initial conditions, specified by $E_{c}$ and $K$, leads to the known melting temperature of $\Gamma_{m}=60$.

[10] D. S. Fisher, B. I. Halperin, and R. Morf, Phys. Rev. B 20, 4692 (1979).

[11] On a lattice with $N$ sites, the dislocation pair $(\vec{b},-\vec{b})$ has three possible orientations and $N$ possible centers. To obtain the numbers $N_{1}(R) \Delta R$ we count possible centers of the partner dislocation $\vec{b}^{\prime}=-\vec{b}$ in the distance regime $R-\Delta R / 2$ and $R+\Delta R / 2$ on a circle about the center of the first dislocation.

[12] K. J. Strandburg, Rev. Mod. Phys. 60, 161 (1988); H. Kleinert, Gauge Fields in Condensed Matter (World Scientific, Singapore, 1989), Vol. II. 